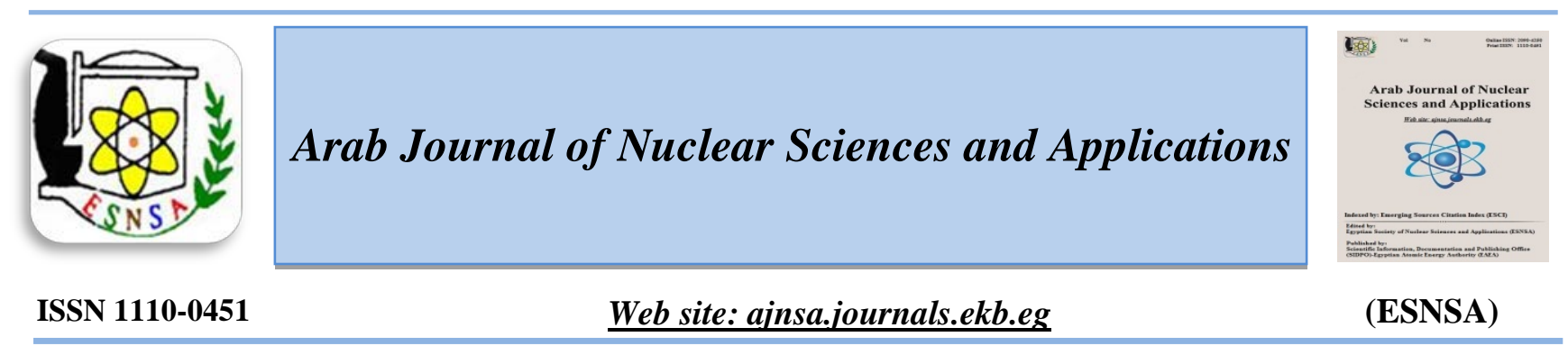

\title{
Evaluation of Gamma Rays Shielding Competence for Bentonite Clay /PVA Polymer Matrix Using MCNPX Code
}

\author{
Fawzy H. Sallam ${ }^{3}$, Ibrahim Z. Hager ${ }^{1}$, Yasser S. Rammah ${ }^{1}$, Hosam A. Othman ${ }^{1}$, Sayed F. Hassan ${ }^{2}$, Eman M. \\ Ibrahim $^{3}$ and Zakaria Ahmed ${ }^{4}$ \\ ${ }^{1}$ Department of Physics, Faculty of Science, Menoufia University, Egypt \\ ${ }^{2}$ Department of Medical and Radiological Research, Nuclear Materials Authority, Egypt \\ ${ }^{3}$ Department of Geochemical Exploration, Nuclear Materials Authority, Egypt \\ ${ }^{4}$ Egyptian Nuclear and Radiological Regulatory Authority, Egypt
}

Received $31^{\text {st }}$ Oct. 2019 Accepted $15^{\text {th }}$ March 202
This Radiation shielding capability in terms of mass and linear attenuation coefficients, half value layer, tenth value layer, and mean free path haven been evaluated for natural bentonite coated with polyvinyl alcohol polymer (PVA) using Monte Carlo simulation (MCNPX) and XCOM program. All data were determined at gamma photons energies 662, 1173 and $1332 \mathrm{keV}$ emitted from point sources of ${ }^{137} \mathrm{Cs}$ and ${ }^{60} \mathrm{Co}$, respectively, and compared with calculated experimental data. XCOM results of mass attenuation coefficients showed better agreement with experimental data in comparison with MCNPX code. The relative deviations between experimental and theoretical mass attenuation coefficients are 4.3, 2.5 and $1.25 \%$ at 662, 1173 and $1332(\mathrm{keV})$ while deviation between simulated and experimental mass attenuation coefficients are $-3.1,-10.96$ and $-10.35 \%$ at the same energies, respectively. The relative deviation between simulated shielding factors HVL, TVL and MFPwith experimental data exhibit the same behavior as mass attenuation coefficient and having average relative deviations percentage equal to 2.5, 13.4 and $13.8 \%$ at the studied energies, respectively.

Keywords: Mass Attenuation Coefficient, Bentonite, PVA Polymer, XCOM, MCNPX code

\section{Introduction}

Wide varieties of materials are being used in radiation protection. The choice of these materials depends on the requirements, application, cost, feasibility, availability, type of radiation, etc. There is always a need to develop material for shielding purposes, which can be used under harsh conditions of nuclear radiation exposure and can act as shielding material [1, 2]. The purpose of radiation shielding is to reduce radiation exposures to the public and workers to an acceptable level [3]. Mineral ores such as magnetite, siderite, barite and limonite ...etc, are popular for producing heavy weight concrete because of economical reasons [4]. Mineral ores can be used as a shield against gamma rays because it contains a variety of light and heavy elements like C, K, S, P, Ca, Mg, $\mathrm{Na}$, etc [5]. Natural bentonite is considered a mineral ore which contains a variety of oxides and can be used as a shield against gamma rays. Polymeric composites containing inorganic additives became the most popular shield against gamma rays and many studies investigated their shielding properties [6-8]. Polymeric nanocomposites have a great potential for improving shielding properties [9-11].

The simulation method for investigation of radiation interaction is found radiologically safer, less time consuming, cost effective and applicable for desired energy of radiation. It is found that Monte Carlo simulation (MCNPX code) is a suitable method for investigation radiation

Corresponding author: thanaasileem@yahoo.com

DOI: 10.21608/ajnsa.2020.18914.1292

(C) Scientific Information, Documentation and Publishing Office (SIDPO)-EAEA 
interaction with materials in the literature [12]. The general purpose of MCNPX code is modeling the interaction of gamma rays with matter and tracking all particles at different energies. It is a full threedimensional and utilizes extended nuclear cross section libraries [13]. Many studies used MCNPX program to demonstrate the effectiveness of nanoparticles in shielding properties [14-16]. Tekin et al, calculated shielding properties of concrete doped with different percentages of $\mathrm{WO}_{3}$ and $\mathrm{PbO}$ using MCNPX simulation at five energies 356, 662, 1173, 1234 and $1333(\mathrm{KeV})$. They concluded that addition of $\mathrm{PbO}$ is more effective than $\mathrm{WO}_{3}$ to reduce radiation dose [17]. Rammah et al investigated six bismuth borate glass samples against gamma rays and calculated its shielding parameters in the range of $0.356-1.33 \mathrm{MeV}$ using MCNPX code. The authors found that replacement of $\mathrm{B}_{2} \mathrm{O}_{3}$ by $\mathrm{Bi}_{2} \mathrm{O}_{3}$ enhance shielding properties of glass [18].

The major aim of the current study is to evaluate the shielding factors for natural bentonite/PVA polymer matrix against gamma rays and this included:

1- Evaluating the mass attenuation coefficients $\left(\mu_{\mathrm{m}}=\mu / \rho\right)$ for the prepared samples at gamma photons energies 662, 1173 and $1332 \mathrm{keV}$ emitted from point sources of ${ }^{137} \mathrm{Cs}$ and ${ }^{60} \mathrm{Co}$, respectively using MCNPX code.

2- The results of simulated $(\mu / \rho)$ have been compared with the theoretical results obtained by WinXCOM program.

3- The simulated and theoretical values were compared with the calculated experimental data.

4- Based on the $(\mu / \rho)$ values, the effective shielding parameters such as LAC, HVL, TVL, and MFP have been calculated.

This study can be very useful for wide applications of natural materials for gamma rays shielding and utilization of standardized geometry of Monte Carlo simulation for medical physics, radiation physics, shielding and radiation protection.

\section{Theoretical background}

The linear attenuation coefficient can be calculated according to The Lambert-Beer law which describes attenuation of a monoenergetic beam as follows:

$$
\mathrm{I}=\mathrm{I}_{0} \mathrm{e}^{-\mu \mathrm{x}}
$$

where $\mathrm{I}$ is the transmitted gamma radiation intensity, $I_{0}$ is the incident gamma radiation intensity, $\mathrm{x}$ is the thickness of the absorbing medium and $\mu$ is the linear attenuation coefficient. For photons in an attenuating medium, the mass attenuation coefficient $(\mu / \rho)$ is given by:

$\mu_{\mathrm{m}}=\mu / \rho=\ln \left(\mathrm{I}_{\mathrm{o}} / \mathrm{I}\right) / \rho \mathrm{X}$

where $\rho$ is the density of the shield material [19]. The theoretical mass attenuation coefficients obtained from XCOM program are calculated using equation (3) at different energies.

$\mu_{\mathrm{m}}=\sum \mathrm{w}_{\mathrm{i}} \mu_{\mathrm{i}}$

where $\mathrm{w}_{\mathrm{i}}$ and $\mu_{\mathrm{i}}$ are percentage by weight and mass attenuation coefficient of the $i^{\text {th }}$ element of the mixture sample [20]. Shielding effectiveness is described in terms of HVL and TVL:

$\mathrm{HVL}=\ln 2 / \mu$

$\mathrm{TVL}=\ln 10 / \mu$

The interaction between two successive interactions; is mathematically the inverse of the linear attenuation coefficient is called mean free path and can be calculated by the equation:

$$
\mathrm{MFP}=1 / \mu
$$

where $\mu$ is the linear attenuation coefficient [21, 22].

\section{Materials and methods}

\section{Characterization of Natural Bentonite}

The properties of the studied cylindrical pellet samples are presented in Table (1). The density of the sample matrix is calculated using Archimedes principle using xylene as an immersion liquid at room temperature. The measurement accuracy was approximately $\pm 0.015 \mathrm{~g} / \mathrm{cm}^{3} \quad$ [24-26]. The chemical composition of the bentonite/PVA matrix sample used in material card of MCNPX code is presented in Table (2). The PVA polymer represents $10 \%$ weight from the all weight of the samples. The percentages of each element in bentonite clay/PVA polymer by weight that used in material card of MCNP code is given in Table (3). 
Table (1): The properties of bentonite/PVA matrices samples

\begin{tabular}{l|llll}
\hline Sample & $\begin{array}{l}\text { Pellet thickness } \\
(\mathbf{c m})\end{array}$ & $\begin{array}{l}\text { Pellet diameter } \\
(\mathbf{c m})\end{array}$ & $\begin{array}{l}\text { Pellet density } \\
\left(\mathrm{g} / \mathrm{cm}^{3}\right)\end{array}$ \\
\hline & 0.5 & & \\
$\begin{array}{l}\text { Natural } \\
\text { bentonite/ } \\
\text { polymer }\end{array}$ & 1 & 2.2 & 1.48 \\
\hline
\end{tabular}

Table (2):Chemical composition of the bentonite/PVA sample.

\begin{tabular}{l|lllllllllll}
\hline Oxides (\%) & $\mathrm{SiO}_{2}$ & $\mathrm{Al}_{2} \mathrm{O}_{3}$ & $\mathrm{P}_{2} \mathrm{O}_{5}$ & $\mathrm{TiO}_{2}$ & $\mathrm{Fe}_{2} \mathrm{O}_{3}$ & $\mathrm{CaO}$ & $\mathrm{MgO}$ & $\mathrm{Na}_{2} \mathrm{O}$ & $\mathrm{K}_{2} \mathrm{O}$ & $\mathrm{H}_{2} \mathrm{O}$ & $\mathrm{C}_{2} \mathrm{H}_{4} \mathrm{O}$ \\
\hline $\begin{array}{l}\text { Natural } \\
\text { Bentonite }\end{array}$ & 27 & 12.2 & 0.31 & 0.31 & 2.9 & 13.7 & 3.63 & 1.3 & 0.6 & 28.05 & 10 \\
\hline
\end{tabular}

Table (3): The percentages of the atomic composition for bentonite clay/PVA matrix

\begin{tabular}{l|lllllllllllll}
\hline Element & $\mathrm{Si}$ & $\mathrm{Al}$ & $\mathrm{P}$ & $\mathrm{Ti}$ & $\mathrm{Fe}$ & $\mathrm{Ca}$ & $\mathrm{Mg}$ & $\mathrm{Na}$ & $\mathrm{K}$ & $\mathrm{H}$ & $\mathrm{O}$ & $\mathrm{C}$ \\
\hline Atomic number & 14 & 13 & 15 & 22 & 26 & 20 & 12 & 11 & 19 & 1 & 8 & 6 \\
\hline $\begin{array}{l}\text { Atomic } \\
\text { composition (\%) }\end{array}$ & 12.5 & 6.5 & 0.14 & 0.2 & 2.03 & $\mathbf{9 . 8}$ & 2.2 & \multirow{2}{*}{0.96} & 0.5 & 4.06 & 55.66 & 5.45 \\
\hline
\end{tabular}

Natural bentonite is considered a mineral ore as it contains several elements in the form of oxides (Table 2). Therefore, it can be used as a shield material for gamma rays. The sample has a water content and organic matter of $28.05 \%$ from all sample contents (loss of ignition value). Silicon oxide and calcium oxide represent the highest oxide contents in the chemical analysis of the studied sample. Polymeric composites become the most popular shield against gamma rays because they protectthe shielding material from environmental conditions, also they prevent nuclear waste leakage through porosity of bentonite clay. The chemical composition of the PVA polymer is $\mathrm{C}_{2} \mathrm{H}_{4} \mathrm{O}$.

\section{MCNPX Code}

MCNPX is a simulation program for radiation transport and modeling of the interaction between radiation and materials at different energies [23]. The samples consist of natural bentonite coated by polyvinyl alcohol polymer (PVA) as a matrix. The PVA polymer represents $10 \%$ weight from the all weight of the samples. The samples are modeled in acylindrical pellet geometry. The cylindrical sample and planer source are set inside a cylindrical collimator with inside cylindrical gap of $2.2 \mathrm{~cm}$ thickness. The diameter of the sample also is $2.2 \mathrm{~cm}$. The distance between source and sample is $1 \mathrm{~cm}$. A collimated monoenergetic narrow beam gamma ray transmits through the sample and hit the detector. The distance between the collimator and the detector is $0.1 \mathrm{~cm}$, while the distance between sample and the detector varies according to the sample thickness. When sample thickness is $0.5 \mathrm{~cm}$, the distance between sample and the detector is $2.1 \mathrm{~cm}(0.1 \mathrm{~cm}$ is the space between collimator and detector while the distance from sample and the end of collimator is $2 \mathrm{~cm}$ ). The geometry of the simulation system is shown in the Fig. (1).

A one cylindrical with $3 " \times 3 " \mathrm{NaI}(\mathrm{Tl})$ of crystal height and diameter of $7.62 \mathrm{~cm}$. The all geometry simulation is inside $55 \mathrm{~mm}$ thick lead shield to isolate the all geometry from external radiation (cosmic rays and earth crust radiation). The geometry of the simulation is set up as experimental conditions. Tally $\left(\mathrm{F}_{4}\right)$ has been used for obtaining simulation data. Simulation is calculated using one million histories and all simulated results were reported with error $\leq 0.1 \%$. In this work, each surface must define in input file in terms of geometric dimension and coordinates also each cell must defined in terms of material content and density of that material. The monoenergetic energies used in the simulation are 662, 1173 and $1332 \mathrm{keV}$ produced by ${ }^{137} \mathrm{Cs}$ and ${ }^{60} \mathrm{Co}$. These energies are the same as used in the experimental conditions for comparison purpose. 


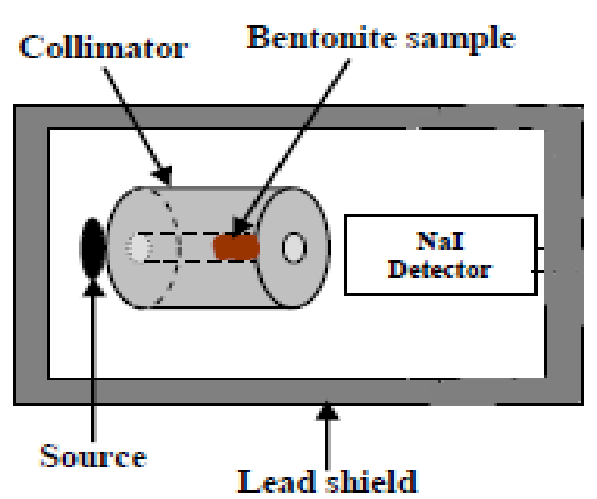

Fig.(1): Geometry of the modeled configuration

\section{Validation}

Applying the Monte Carlo method is the one of the best solutions for the investigation of different complex materials behavior since experimental duplication of investigation is quite complicated. So, it is more suitable to apply some numerical methods such as Monte Carlo. In this paper, a validation for input code was performed. On the other hand, XCOM program was also used to calculate the gamma ray mass attenuation coefficients of the studied shielding materials. $\mathrm{XCOM}$ program is a user friendly calculation program and input parameter specifications are quite flexible and easy to access. In the XCOM program, firstly, shielding material types were defined by their elemental mass fractions, which are totally the same as in MCNPX Monte Carlo code input. Secondly, the gamma ray energies have been defined. The attenuation coefficients of the selected materials were finally calculated by the program.

\section{Results And Discussion}

Mass attenuation coefficient is a fundamental property of a material for photon interaction to represent interaction and shielding effectiveness. Mass attenuation coefficient of an element is found to be constant at a particular photon energy, whereas mass attenuation coefficient of a compound or mixture depends upon composition of elements. The linear attenuation coefficient, half-value layer thickness, tenth-value layer thickness and effective atomic number are derived parameters from mass attenuation coefficient [12].

\section{Linear and Mass Attenuation Coefficients}

Shielding factors of a natural bentonite/PVA sample has been calculated experimentally using the same geometry used in MCNPX code at the same energies. Equation (1) of Beer-Lambert is used in linear attenuation coefficient determination. By plotting the relation between Ln (Io/I) versus sample thickness (x), the slope is equal to the linear attenuation coefficient value. The obtained data for natural bentonite/PVA samples is given in Table (4) and drawn in Fig.(2).

Table (4):Measured gamma photons with and without natural bentonite/PVA samples

\begin{tabular}{|c|c|c|c|}
\hline $\begin{array}{l}\text { Energy (KeV) } \\
\text { Thickness (cm) }\end{array}$ & 662 & 1173 & 1332 \\
\hline $\mathbf{0}$ & 18189 & 2918 & 2276 \\
\hline 0.629 & 17219 & 2784 & 2231 \\
\hline 1.079 & 16643 & 2692 & 2153 \\
\hline 1.661 & 16024 & 2605 & 2094 \\
\hline 2.012 & 15619 & 2513 & 2018 \\
\hline 3 & 13503 & 2301 & 1848 \\
\hline 3.55 & 12440 & 2143 & 1750 \\
\hline
\end{tabular}




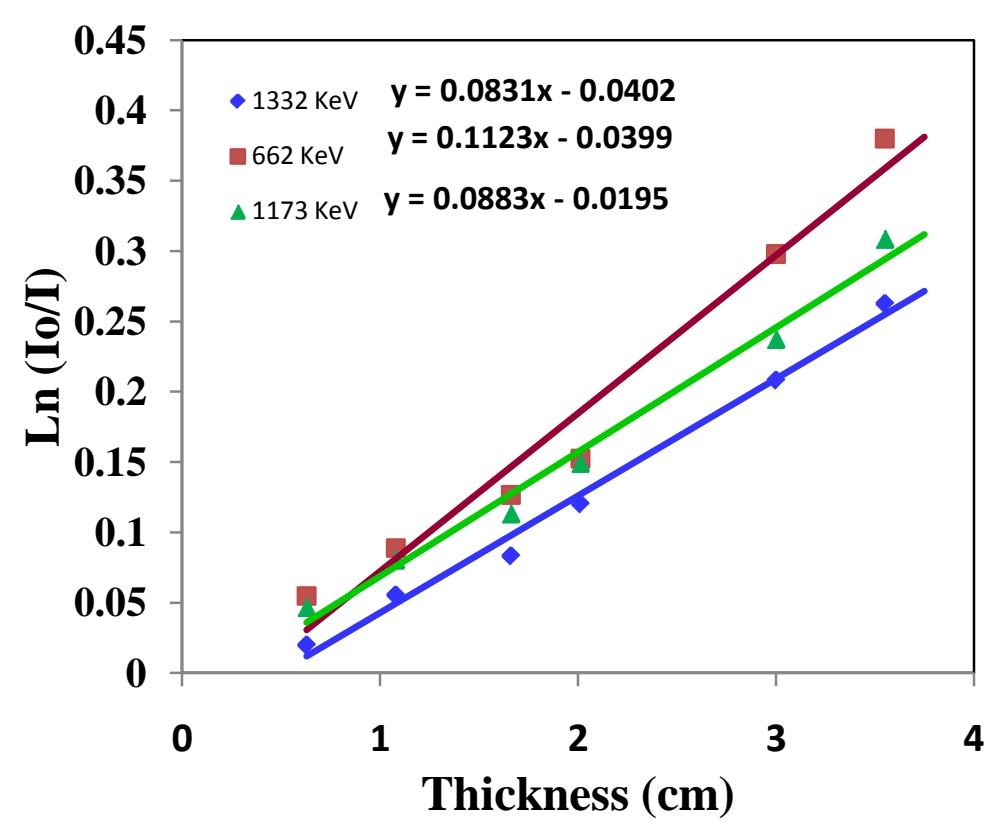

Fig.(2):Gamma transmission through natural bentonite/PVA sample at different thicknesses and different energies

Shielding parameters of natural bentonite/PVA samples $\left(\mu, \mu_{\mathrm{m}}, \mathrm{HVL}\right.$, TVL and mfp) calculated experimentally using equations $1,3,4,5$ and 6 are summarized in Table (5). The density of bentonite/PVA samples is $1.48 \mathrm{~g} / \mathrm{cm}^{3}$ which was measured using Archimedes principle.

MCNPX simulation has been used for linear and mass attenuation coefficients calculations of bentonite/PVA samples with different thicknesses. The linear and mass attenuation coefficients for attenuator samples were calculated for the three different gamma energies 662, 1173 and $1332 \mathrm{keV}$ and shown in Table (6) and Fig.(3).It is found that the linear and mass attenuation coefficients of the studied samples are decreasing with increase in photon energy. This variation of mass attenuation coefficients can be explained using the fundamental photon interaction process of photoelectric effect, Compton scattering and pair production for low-, intermediate- and high energy photons, vary with atomic number of elements of compositions. Additionally, at low photon energy, photoelectric interaction is the dominance, and is the reason for mass attenuation coefficient reduction (662 keV) while at $1173 \mathrm{keV}$ and 1332 $\mathrm{keV}$ Compton scattering and pair production are dominant [27]. The variations of average linear and massattenuation coefficients versus photon energy (keV) are illustrated in Fig.(4). The mass and linear attenuation coefficients exhibit the same behavior. 
Table (5): Experimental calculation of shielding parameters for bentonite/PVA sample

\begin{tabular}{l|lllll}
\hline Shielding parameter & $\mu\left(\mathrm{cm}^{-1}\right)$ & $\mu_{\mathrm{m}}\left(\mathrm{g} / \mathrm{cm}^{2}\right)$ & HVL $(\mathbf{c m})$ & TVL $(\mathbf{c m})$ & Mfp (cm) \\
Energy (KeV) & 0.112 & 0.076 & 6.188 & 20.55 & 8.92 \\
662 & 0.088 & 0.059 & 7.876 & 26.16 & 11.36 \\
1173 & 0.083 & 0.056 & 8.351 & 27.74 & 12.04 \\
\hline
\end{tabular}

Table (6): Simulated linear $\left[\mu\left(\mathrm{cm}^{-1}\right)\right]$ and mass $\left[\mu_{\mathrm{m}}\left(\mathrm{cm}^{2} / \mathrm{g}\right)\right]$ attenuation coefficients of bentonite/PVA polymer samples

\begin{tabular}{l|ll|ll|ll}
\hline \multirow{2}{*}{ Energy(keV) } & 662 & & 1173 & & 1332 \\
\cline { 2 - 7 } Thickness & $\boldsymbol{\mu}$ & $\boldsymbol{\mu}_{\mathrm{m}}$ & $\boldsymbol{\mu}$ & $\boldsymbol{\mu}_{\mathrm{m}}$ & $\boldsymbol{\mu}$ & $\boldsymbol{\mu}_{\mathrm{m}}$ \\
\hline $\mathbf{0 . 5}$ & $\mathbf{0 . 1 1 4}$ & $\mathbf{0 . 0 7 6 1 9}$ & $\mathbf{0 . 0 8 2 6}$ & $\mathbf{0 . 0 5 5 8 2}$ & $\mathbf{0 . 0 7 7 5}$ & $\mathbf{0 . 0 5 3 1 2}$ \\
1 & $\mathbf{0 . 1 1 0 8 6}$ & $\mathbf{0 . 0 7 4 8 1}$ & $\mathbf{0 . 0 8 0 4}$ & $\mathbf{0 . 0 5 4 3 3}$ & $\mathbf{0 . 0 7 5 1 1}$ & $\mathbf{0 . 0 5 1 4 4}$ \\
1.5 & $\mathbf{0 . 1 0 9 3}$ & $\mathbf{0 . 0 7 3 7 6}$ & $\mathbf{0 . 0 7 7 3 5}$ & $\mathbf{0 . 0 5 2 2 6}$ & $\mathbf{0 . 0 7 3 5 8}$ & $\mathbf{0 . 0 5 0 7 7}$ \\
2 & $\mathbf{0 . 1 0 7 0 3}$ & $\mathbf{0 . 0 7 2 2 2}$ & $\mathbf{0 . 0 7 5 0 6}$ & $\mathbf{0 . 0 5 0 7 2}$ & $\mathbf{0 . 0 7 1 5 1}$ & $\mathbf{0 . 0 4 9 0 7}$ \\
2.5 & $\mathbf{0 . 1 0 5 5 5}$ & $\mathbf{0 . 0 7 1 2 3}$ & $\mathbf{0 . 0 7 3 2 9}$ & $\mathbf{0 . 0 4 9 5 1}$ & $\mathbf{0 . 0 6 7 7}$ & $\mathbf{0 . 0 4 6 7 9}$ \\
\hline Average & $\mathbf{0 . 1 0 9 3 4}$ & $\mathbf{0 . 0 7 3 6 4}$ & $\mathbf{0 . 0 7 7 7 4}$ & $\mathbf{0 . 0 5 2 5 2 8}$ & $\mathbf{0 . 0 7 4 0 8}$ & $\mathbf{0 . 0 5 0 2}$ \\
\hline
\end{tabular}

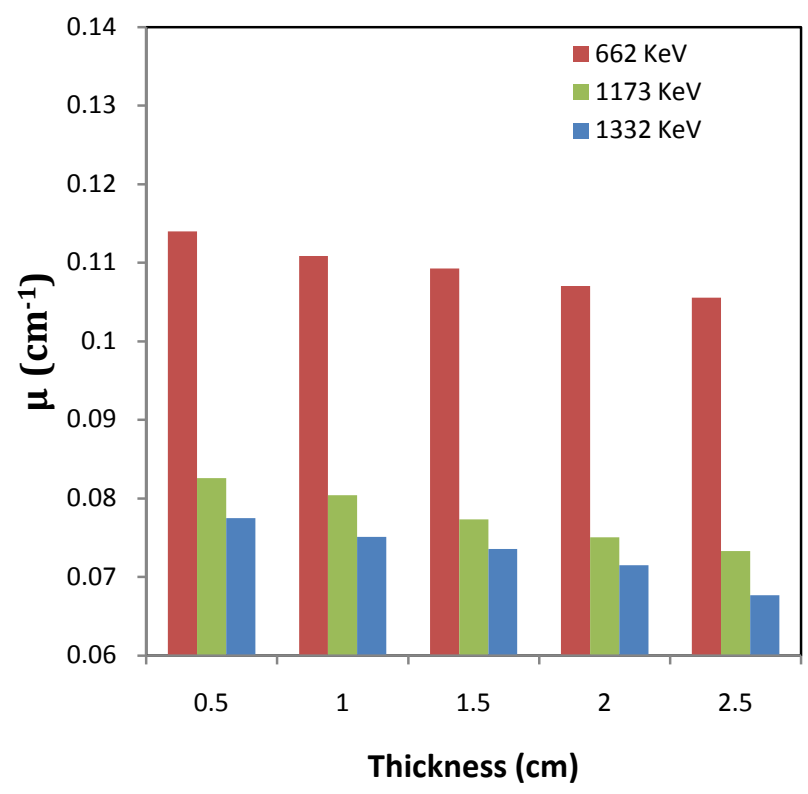

Fig.(3): Linear ( $\mu$ )and mass $\left(\mu_{m}\right)$ attenuation coefficients of bentonite/PVA samples estimated using MCNPX at different energies 
The $\mu_{\mathrm{m}}$ values for bentonite/PVA samples were calculated using the MCNPX and XCOM codes for photon energies 662, 1173 and $1332 \mathrm{keV}$. The simulation results were compared with the experimental results (Table 5). The simulated MCNPXand XCOM results of $\mu_{\mathrm{m}}$ are plotted together with the previous experimental results as shown in Fig.(5)at the aforementioned photon energies.It can be seen from Fig.(5)that thereis a satisfactory agreement between the theoretical (XCOM) method and experimental results. However, the $\mu_{\mathrm{m}}$ values calculated by MCNPX were found to be slightly lower than the experimental results at different photon energies. The discrepancies between the simulated $\mu_{\mathrm{m}}$ values and experimental data can be attributed to deviation from narrow beam geometry in the source-detector arrangement.

In Fig.(6), the relative deviation (RD), differences between simulation and theoretical results with experimental data of mass attenuation coefficients for 662, 1173 and $1332 \mathrm{keV}$ gamma ray energies are plotted for MCNPX code and XCOM program using Eq.(7) [28]:

$\mathrm{RD}=($ Theoretical - experimental $) \quad \mathrm{x} \quad 100 /$ Experimental(7)

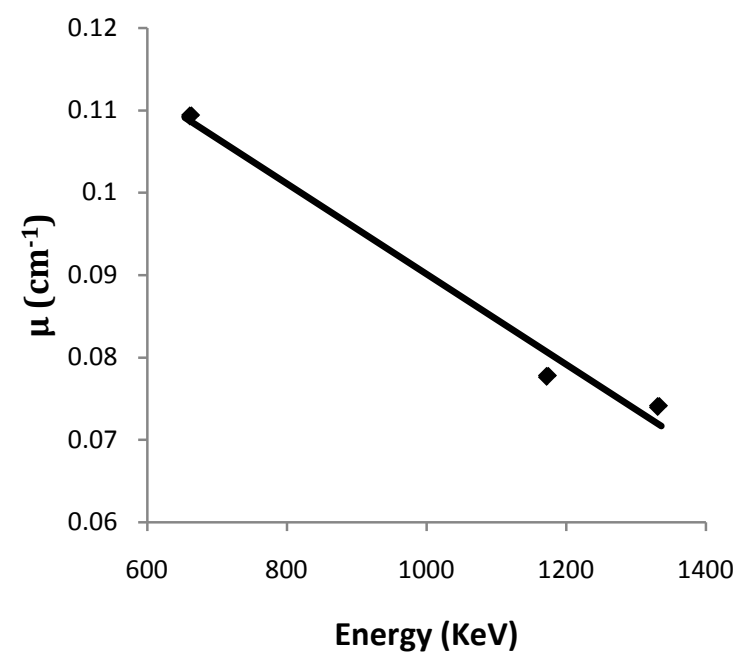

Fig.(4): Variations of average linear and mass attenuation coefficients with gamma energies

Table (7): Comparison between average simulated (MCNPX-code), theoretical (XCOM) and available experimental (Exp.) mass attenuation coefficients data at different energies

\begin{tabular}{llll}
\hline Energy (keV) & MCNPX & XCOM & Exp. \\
\hline 662 & 0.07364 & 0.0793 & 0.076 \\
1173 & 0.05253 & 0.0605 & 0.059 \\
1332 & 0.0502 & 0.0567 & 0.056 \\
\hline
\end{tabular}




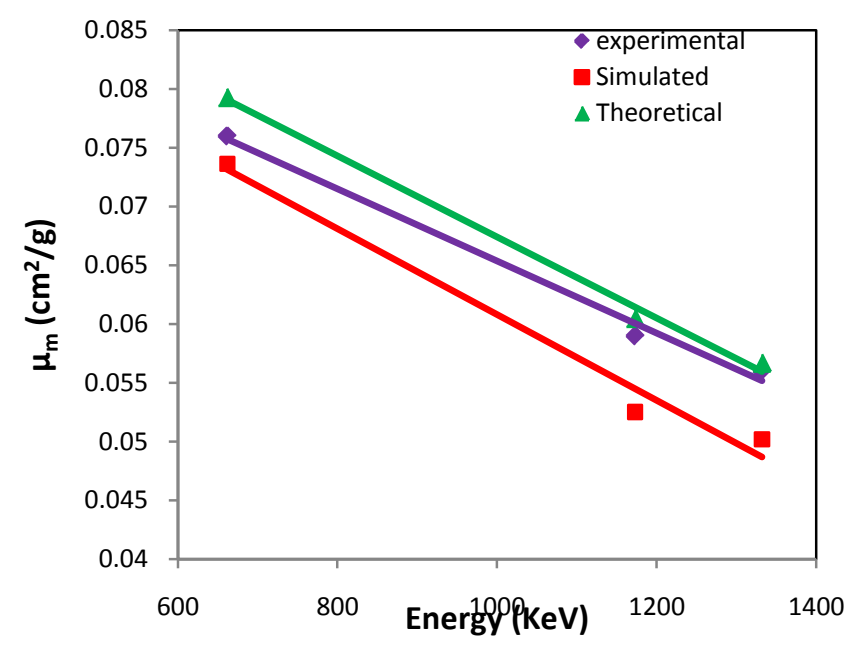

Fig.(5):Comparison between average simulated, theoretical and experimental mass attenuation coefficients at different energies

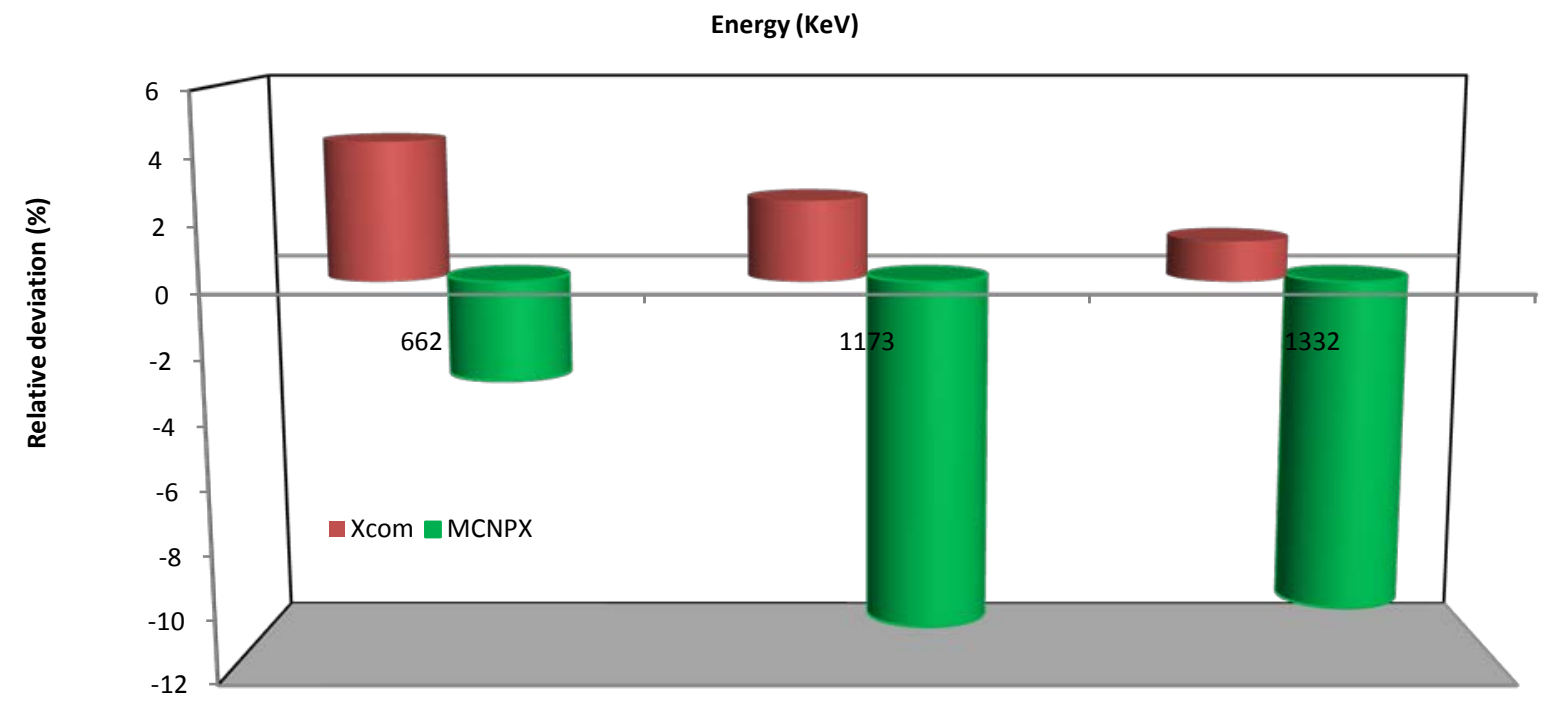

Fig.(6): Difference (\%) between experimental data and MCNPX and XCOM results

The relative deviation $(\mathrm{RD})$ values are $-3.1 \%$, $10.96 \% \&-10.35 \%$ and $4.3 \%, 2.5 \% \& 1.25 \%$ for MCNPX and XCOM results relative to experimental mass attenuation coefficient at 662, 1173 and $1332 \mathrm{keV}$ photon energies, respectively, for all samples. It was found from Fig.(6) that approximately a good agreement was observed between experimental and theoretical values and the discrepancies are not being considered to be large, because the differences are in the magnitude 
of the reported experimental errors which is less than $5.05 \%$ in average [29]. There is a little difference between experimental and simulated mass attenuation coefficient reaching $11 \%$. It should be noted that the difference between simulated data and both experimental and theoretical data are attributed to the difference between the employed techniques and database for each method and also to the utilized extended nuclear cross section libraries. Natural bentonite/PVA polymer matrix sample is a promising mixture, as its mass attenuation coefficient represents $69 \%$ percentage in comparison with theoretical lead value 0.1101 $\mathrm{cm}^{2} / \mathrm{g}$ [20]. The natural bentonite represents a mixture of elements as it consists of a variety of mineral oxides that effective in shielding gamma rays.

Half Value Layer (HVL), Tenth Value Layer (TVL) and Mean Free Path (MFp)

It is more useful in the radiation fields to express attenuation of gamma rays in terms of half value layer (HVL), which is defined as the thickness from the absorber material that reduces initial intensity of gamma rays $50 \%$ from its value. Another factor plays the same role as half value layer is the tenth value layer (TVL) which defined as the thickness from the absorber material that reduces initial intensity of gamma rays to one tenth of its value. Both HVL and TVL describe the effectiveness of the shielding material. The mean free path (MFP) parameter is one of the basic quantities required for gamma rays shielding and are calculated for bentonite/PVA matrix sample at 662, 1173 and $1332 \mathrm{keV}$ using MCNPX code. The results of linear attenuation obtained from MCNPX program were used for obtaining the value of HVL, TVL and MFP using equations (4), (5) and (6), respectively. The calculated HVL, TVL and MFP values are listed in Table (8) and drawn in Fig.(7). These Figures show that the HVL, TVL and MFPvalues of the samples increase with the increase in thethickness of the sample and the incident photon energy. The average HVL, TVLand MFPvalues are (6.343, 8.933 and 9.505 $\mathrm{cm}),(21.072,29.675$ and $31.574 \mathrm{~cm})$ and $(9.151$, 12.887 and $13.712 \mathrm{~cm}$ ) for 662, 1173 and 1332 $\mathrm{keV}$, respectively.

Table (8): HVL, TVLandMFP(cm) values of bentonite/PVA polymer samples at different energies

\begin{tabular}{|c|c|c|c|c|c|c|c|c|c|}
\hline \multirow{2}{*}{$\begin{array}{l}\text { Energy(keV) } \\
\text { Thickness }\end{array}$} & \multicolumn{3}{|l|}{662} & \multicolumn{3}{|l|}{1173} & \multicolumn{3}{|l|}{1332} \\
\hline & HVL & TVL & MFP & HVL & TVL & MFP & HVL & TVL & MFP \\
\hline 0.5 & 6.08 & 20.198 & 8.771 & 8.391 & 27.876 & 12.106 & 8.943 & 29.710 & 12.903 \\
\hline 1 & 6.252 & 20.770 & 9.020 & 8.621 & 28.639 & 12.437 & 9.228 & 30.656 & 13.313 \\
\hline 1.5 & 6.342 & 21.066 & 9.149 & 8.961 & 29.768 & 12.928 & 9.420 & 31.293 & 13.590 \\
\hline 2 & 6.476 & 21.513 & 9.343 & 9.234 & 30.676 & 13.322 & 9.693 & 32.199 & 13.984 \\
\hline 2.5 & 6.567 & 21.815 & 9.474 & 9.457 & 31.417 & 13.644 & 10.238 & 34.011 & 14.771 \\
\hline Average & 6.343 & 21.072 & 9.151 & 8.933 & 29.675 & 12.887 & 9.505 & 31.574 & 13.712 \\
\hline
\end{tabular}




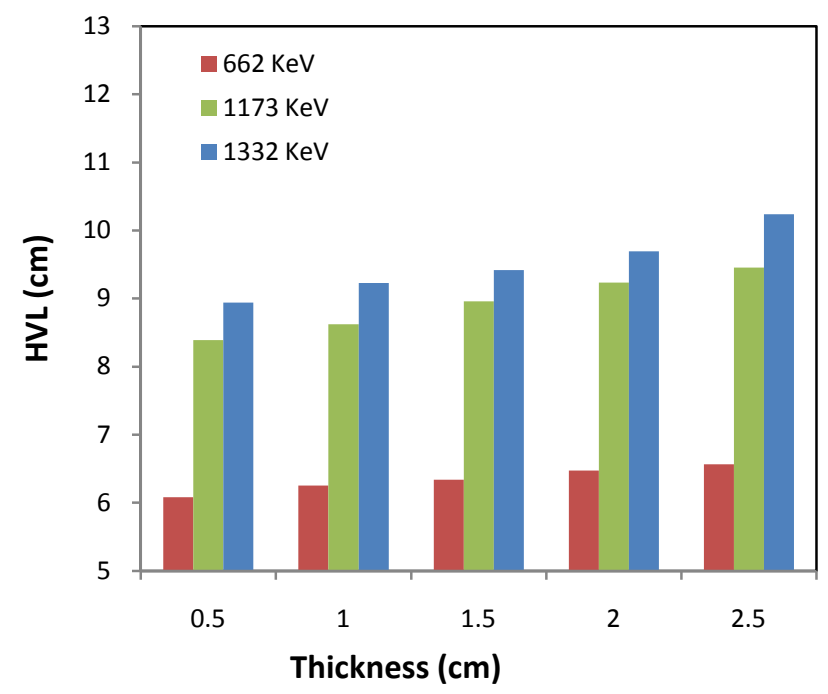

Fig.(7): HVL, TVL and mfpvariations with thickness at different photon energies

Table (9): Comparison between simulated (MCNPX) and experimental (Exp.) shielding factors (HVL, TVL and MFP)

\begin{tabular}{llllllll}
\hline \multirow{2}{*}{ Energy (keV) } & HVL & \multicolumn{3}{c}{ TVL } & & MFP & \multicolumn{2}{c}{$\begin{array}{l}\text { Relative } \\
\text { Deviation } \\
(\%)\end{array}$} \\
\cline { 2 - 9 } & MCNPX & Exp. & MCNPX & Exp. & MCNPX & Exp. & (\%) \\
\hline 662 & 6.343 & 6.188 & 21.072 & 20.55 & 9.151 & 8.92 & 2.5 \\
1173 & 8.933 & 7.876 & 29.675 & 26.16 & 12.887 & 11.36 & 13.4 \\
1332 & 9.504 & 8.351 & 31.574 & 27.74 & 13.712 & 12.04 & 13.8 \\
\hline
\end{tabular}

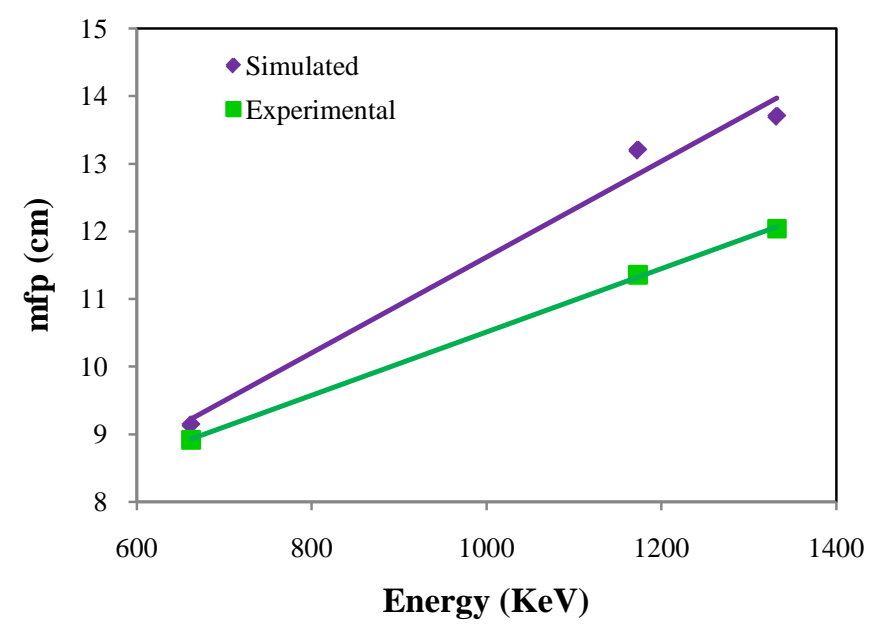

Fig.(8) Comparison between simulated and experimental values for HVL, TVL and MFP at different energies 
The comparisons between simulated and experimental data for shielding factors (HVL, TVL and mean free path) at different energy values are given below in Table( 9) and Fig.(8). The comparison between shielding factors (HVL, TVL and MFP) showed that the three factors have the same behavior where they increase with the increasing of photon energy for experimental and simulated data. The shielding factors (HVL, TVL and MFP) have the same relative deviations between experimental and simulated data and there average values are 2.5, 13.4 and 13.8 (\%) at 662, 1173 and 1332 (keV), respectively.

\section{Conclusion}

Radiation shielding parameters (mass attenuation coefficient, linear mass attenuation coefficient, HVL, TVL and mean free path) were calculated for bentonite clay /PVA polymer matrix sample at 662, 1173 and $1332 \mathrm{keV}$ using MCNPX program and XCOM program and were compared with the experimental data. It was found that theoretical mass attenuation coefficient is in a very good agreement with the experimental data and have relative deviation of 4.3, 2.5 and $1.25 \%$ while, the calculated shielding parameters (mass, linear, HVL, TVL and MFP) from MCNPX program has a little difference with experimental data. The comparison showed relative deviations of -3.1, 10.96 and -10.35 (\%) at 662, 1173 and 1332 (keV), respectively, for mass attenuation coefficient while the average relative deviations of other shielding parameters (HVL, TVL and MFP) of 2.5, 13.4 and $13.8(\%)$ at the same energies. The data obtained from MCNPX program is a reliable data for bentonite/PVA sample within $\pm 13 \%$ in comparison with experimental data and $\pm 5 \%$ in comparison with theoretical data. The difference percentage in comparison with different techniques, is due to different database and different utilized extended nuclear cross section libraries.

\section{References}

1-Krocher J. F.,Browman R.E. (1984),Effects of radiation on materials and components. Reinhold (Eds.), New York.

2-Singh N., Singh K.J., Singh K., Singh H. (2004),Comparative study of lead borate and bismuth lead borate glass systems as gamma-radiation shielding materials. Nucl. Instru. Meth. Physi. Rese. B, 225(3), 305-309.

3-Glasgow G.P. (2006), Structural shielding design and evaluation for megavoltage $\mathrm{X}$ - and gamma-ray radiotherapy facilities.Med. Phys., 2006 Sep, 33(9), 3578.

4-BashterI.I., Abdo A.E.,MakariousA.S. (1996), $\underline{A}$ comparative study of the attenuation of reactor thermal neutrons in different types of concrete. Ann. Nucl. Ener. 23(14), 1189-1195.

5-Laxman C., Dayanand R. (2012), Attenuation coefficient of soil samples by gamma ray energy, Res.J.Rece. Sci., 1(9), 41-48.

6-Abdo A.E.S., Ali, M.A.M., Ismail M. R. (2003),Natural fibre high-density polyethylene and lead oxide composites for radiation shielding. Radi.Physi. Chem., 66(3), 185-195.

7-Lee B. J., McDonald A. G., James B. (2001), Influence of fiber length on the mechanical properties of wood fiber/polypropylene prepreg sheets. Mater. Resea. Innova., 4(2-3), 97-103.

8-Abdo A.E. S., El-SarrafM.A., GaberF.A. (2003),Utilization of ilmenite/epoxy composite for neutrons and gamma rays attenuation. Ann.Nucl. Ener., 30(2), 175-187.

9-Atta E.R, Zakaria K.M.,Madbouly A.M. (2015),Study on polymer clay layered nanocomposites as shielding materials for ionizing radiation, Inter. J.Rece. Sci. Rese., 6(5), 4263-4269.

10-El-Tantawy F., Kamada K., Ohnabe H. (2002), On the 'curiosity' of electrical self-heating, static charge and electromagnetic shielding effectiveness from carbon black/aluminium flakes reinforced epoxyresin composites. Poly.Inter., 51, 635-646.

11-11 Kumar R.V., Palchik O., Koltypin Y., Diamant Y., GedankenA. (2002), Sonochemical synthesis and characterization of $\mathrm{Ag}_{2} \mathrm{~S} / \mathrm{PVA}$ and $\mathrm{CuS} / \mathrm{PVA}$ nanocomposite. Ultras. Sono., 9, 65-70.

12-Tekin H. O., Singh V. P., Manici T., Altunsoy E.E. (2017), Validation of MCNPX with Experimental Results of Mass Attenuation Coefficients for Cement, Gypsum and Mixture. J.Radi.Prote.Resea., 42(3), 154-157.

13-MCNPX ${ }^{\mathrm{TM}}$ 2.4.0, RSICC Computer Code Collection. MCNPX User's Manual Version 2.4.0. Monte Carlo N-ParticleTransport Code System for Multiple and High Energy Applications, Los Alamos National Laboratory, Los Alamos, New Mexico (2002).

14-Khadem-Abolfazli M., Mahdavi S.R.,Ataei G. (2015), Studying effects of gold nanoparticle on dose enhancement in megavoltage radiation. J.Biom. Phys.Engi., 5-4, 185-190.

15-Dong Y., Chang S., Zhang H., Ren C., Kang D.M., Dai Y. (2012), Effects of $\mathrm{WO}_{3}$ particle size in $\mathrm{WO}_{3}$ /epoxy resin radiation shielding material. Chin. Phys. Lett., 29, 1-4.

16-Ghorbani M.,Pakravan D. I., Bakhshabadi M.,Meigooni A.S. (2012), Dose Enhancement In Brachytherapy In The Presence Of Gold Nanoparticles: A Monte Carlo Study On The Size Of

Arab J. Nucl. Sci. \& Applic. Vol. 53, No. 2 (2020) 
Gold Nanoparticles And Method Of Modelling. NUKLEONIKA. 57-3, 401-406.

17-Tekin H. O., Sayyed M. I., Altunsoy E. E andManici T. (2017), Shielding properties of $\mathrm{WO}_{3}$ and $\mathrm{PbO}$ on mass attenuation coefficients by using MCNPX code , Digest Journal of Nanomaterials and Biostructures, Vol. 12, No. 3, p. $861-867$.

18-Rammah Y. S., Sayyed M. I., Ali A. A.,Tekin H. O. and El-Mallawany R. (2018),Optical properties and gamma-shielding features of bismuth borateglasses, Applied Physics A 124:832,https://doi.org/10.1007/s00339-018-2252-7

19-El-Fiki S., El Kameesy S. U., Nashar D. E.E., AbouLeila M. A., El-Mansy M. K., Ahmed M. (2015), Influence of bismuth contents on mechanical and gamma ray attenuation properties of silicone rubber composite. Inter. J. Adv. Rese., 3(6), 1035-1039.

20-Berger M. J., Hubbell J.H. (1999), XCOM: Photon cross sections data base, Web Version, 1.2. National Institute of Standards and Technology, Gaithersburg, MD20899, USA. (Originally published as NBSIR 87-3597 “XCOM: Photon Cross Sections on a Personal Computer',), http://physics.nist.gov./xcomS.

21-Akkurt I.,Altindag R.,Gunoglu K.,Sarikaya H. (2012), Photon attenuation coefficients of concrete including marble aggregates. Ann.Nucl. Ener., 43, 56-60.

22-TsoulfaniidisN., (1983), Measurement and detection of radiation, Hemisphere PublishingCorporation: Mcgraw-Hill Book Company, 151-163.

23-TekinH.O., Singh V.P.,Manici T. (2017),Effects of micro-sized and nano-sized $\mathrm{WO}_{3}$ on mass attenuation coefficients of concrete by using MCNPX code, Appl. Radia. Isot., 121, 122-125.

24-Hager I. Z., Rammah Y. S., Othman H.A., Ibrahim, E.M., Hassan S. F., Sallam F.H. (2019), Nanostructured natural bentonite clay coated by polyvinyl alcohol polymer for gamma rays attenuation, Journal of Theoretical and Applied Physics, https://doi.org/10.1007/s40094-019-0332-5.

25-Alam M. N., Miah M.M. H., Chowdhury M. I., Kamal M., Ghose S., Rahman R. (2001), Attenuation coefficients of soils and some building materials of Bangladesh in the energy range 276-1332 keV. Technical note, Appl. Radiat. Isot., 54(6), 973-976.

26-Othman H.A., ElkholyH.S, Hager I.Z. (2016),FTIR of binary lead borate glass: Structural investigation, Journal of Molecular Structure 1106 286-290.

27-Hassan H.E.,BadranH.M.,Aydarous A.,Sharshar T. (2015),Studying the effect of nano lead compounds additives on the concrete shielding properties for $\gamma$ rays. Nucl. Instru. Meth. Phys. Rese. B, 360, 81-89.

28-Bagheri R., Shirmardi S. P., Adeli R. (2017), Study on gamma-ray shielding characteristics of lead oxide, barite and boron ores using MCNP-4C Monte Carlo code and experimental data. J. Test. Eval., 45(6), 2259-2266.

29-Un A., Sahin Y. (2011), Determination of mass attenuation coefficients, effective atomic and electron numbers, mean free paths and kermas for $\mathrm{PbO}$, barite and some boron ores, Nucl. Instrum. Meth., B 269, 13, 1506-1511. 